\title{
Can hellicobacter pylori infection increase the risk of variceal bleeding in patients with liver cirrhosis?
}

\begin{abstract}
Background and study aims: Gastro-oesphageal varices carry high risk of bleeding which may be fatal in some circumstances. Many patients with varices have Helicobacter pylori (H.Pylori) infection in their gastric mucosa. The relationship between H.Pylori infection and the incidence of upper GIT bleeding was not well studied. Our study was aimed to study the role of H.Pylori infection in gastro-oesphageal variceal bleeding.
\end{abstract}

Patients and Methods: The subjects were 392 cirrhotic patients who were admitted to the Zagazig University Hospital to treat their gastro- oesphageal varices, consisted of 190 with upper GIT bleeding (bleeder group) and 202 with non bleeding episodes but came for prophylactic purposes either primary or secondary prevention (non bleeder group). For the diagnosis of $H$. Pylori infection, rapid urease test was done for endoscopic gastric biopsies.

Results: Gastro-oesphageal variceal bleeding was seen in $31.6 \%(\mathrm{n}=60)$ of the H.Pylori infected patients and in $68.4 \%(\mathrm{n}=130)$ of the no infected patients (total number was 190 patients) $(\mathrm{P}<0.001)$. The H.Pylori infection was associated with low incidence of bleeding in patients with gastro-eosphageal varices.

Conclusion: These results suggest that H.pylori infection may have a protective effect against gastro-esophageal variceal bleeding.

Keywords: hellicobacter pylori, gastro oesphageal varices, upper git bleeding
Volume 4 Issue I - 2018

\author{
Hesham Attia Abd ELGhany,Amr Talaat EL \\ Hwary, Ahmed Fathy Goma \\ Department of Internal Medicine, Zagazig University, Egypt
}

Correspondence: Hesham Attia Abd ELGhany, Internal Medicine Department, Zagazig university Hospital, Egypt, Tel 010 9096 950I, Email gom3a20042000@yahoo.com

Amr Talaat EL Hwary, Internal Medicine DepartmentHepatology Division, Zagazig University, Zagazig Egypt, Tel 010 9096950 I, Email gom3a20042000@yahoo.com

Received: September 27, 2017 | Published: February 02, 2018
Abbreviations: iNOS, inducible nitric oxide synthase; PPI, proton pump inhibitors; ALT, alanine aminotransferase; AST, aspartate aminotransferase; EVL, endoscopic variceal ligation; EIS, endoscopic injection sclerotherapy; SPSS, statistical package for social science; MALT, mucosa-associated lymphoid tissue

\section{Introduction}

H.Pylori is a gram-negative bacterium which lives in gastric mucosa, is the main cause of gastritis type B, ${ }^{1}$ peptic ulcer disease and Mucosa-associated lymphoid tissue (MALT) lymphoma. ${ }^{2}$

Portal hypertension in patients with cirrhosis is the cause of many dangerous complications, such as hepatic encephalopathy, ascites, and gastro-oesophageal variceal bleeding. Gastro-oesophageal variceal bleeding is the cause of more than one-third of deaths in patients with liver cirrhosis. ${ }^{3}$ H.Pylori infection has a role in some diseases as peptic ulcer $^{4}$ portal hypertensive gastropathy ${ }^{5}$ and hepatic encephalopathy. ${ }^{6}$ However, the role of H.Pylori infection is not well studied in cirrhotic patients with gastro-oesphageal variceal bleeding. The aim of our study was to assess and clarify the effect and the correlation between H.Pylori infection and bleeding from gastro-oesophageal varices in patients with liver cirrhosis and portal hypertension.

\section{Patients and methods}

This cross-sectional study was conducted in the Gastroenterology, Hepatology and Endoscopy unit of Internal Medicine department, Zagazig University Hospital from December 2015 to may 2017. Informed consent and agreement of ethical committee was obtained from all patients.

A total number of 392 patients with gastro-oesphageal varices were admitted to the hospital for treatment, the patients were classified into two groups:

a. Group A: 190 patients with active upper GIT bleeding (bleeding group).

b. Group B: 202 patients without active upper GIT bleeding for elective endoscopy to prevent recurrent bleeding (non-bleeding group).

\section{Inclusion criteria}

Subjects included in this study were cirrhotic patients, with age group 25-65years old, both males and females.

\section{Exclusion criteria}

i. Patients with known peptic ulcer disease.

ii. Those who receiving anti-ulcer treatment in the last three months and still receiving proton pump inhibitors (PPI) or $\mathrm{H} 2$ receptor blockers.

iii. Those previously diagnosed to have H.Pylori infection and those who had undergone or were currently undergoing H.Pylori eradication.

\section{Methods}

All the included patients will be subjected to the following: Full history taking, full clinical examination, laboratory investigations including: $\mathrm{CBC}$, liver function tests as prothrombin time, serum albumin, Alanine Aminotransferase (ALT), Aspartate Aminotransferase (AST), total and direct bilirubin, kidney function tests including blood urea and creatinine. 


\section{Abdominal ultrasonography, upper gastrointestinal Endoscopy}

Endoscopic variceal ligation (EVL) or endoscopic injection sclerotherapy (EIS) with 5\% ethanolamine oleate was performed for securing oesophageal varices and injection with histoacryl was performed for securing gastric varices.

A. Child-Pugh classification was done on admission by using parameter of serum bilirubin, serum albumin, prothrombin concentration, hepatic encephalopathy and ascites.

B. Rapid urease test was done for gastric biopsies to diagnose H.Pylori infection by using (rapid urease test kits, Kimberlyclark; Ballard, USA).

\section{Statistical analysis}

a) The collected data were computerized and statistically analyzed using SPSS program (Statistical Package for Social Science) version 20. Qualitative data were represented as frequencies and relative percentages.

b) Chi square test $\left(\chi^{2}\right)$ and Fisher exact was used to calculate difference between qualitative variables as indicated. Quantitative data were expressed as mean \pm SD (Standard deviation). Independent $\mathrm{T}$ test was used to calculate difference between quantitative variables in two groups in normally distributed data. The significance Level for all above mentioned statistical tests done. The threshold of significance is fixed at $5 \%$ level (P-value) $* \mathrm{P}$ value of $>0.05$ indicates non-significant results. ${ }^{*} \mathrm{P}$ value of $\leq 0.05$ indicates significant results.

\section{Results}

Two hundred and fifty nine of our cases were males with a percentage of $66 \%$ and one hundred and thirty three patients were females with a percentage of $34 \%$. Among of them one hundred and ninety patients presented with upper GIT bleeding with a percentage of $48.4 \%$ and two hundred and two patients were non bleeders with percentage of 51.6\% (admitted for elective upper endoscopy). According to Child-Pough scoring system: The patients were divided as follow shown in Table 1 .

Table I Classification of the groups according to child scoring system

\begin{tabular}{lllll}
\hline \multirow{2}{*}{ Child score } & \multicolumn{2}{l}{ Group A(190) } & \multicolumn{2}{l}{ Group B(202) } \\
\cline { 2 - 5 } & Number & Percentage & Number & Percentage \\
\hline A & 60 & $31.50 \%$ & 53 & $26.20 \%$ \\
B & 113 & $59.40 \%$ & 114 & $56.40 \%$ \\
C & 17 & $9.10 \%$ & 35 & $17.40 \%$ \\
\hline
\end{tabular}

By abdominal ultrasound 43 patients with a percentage of $11 \%$ having hepatic focal lesions, 313 patients with a percentage of $79.8 \%$ having splenomegaly and 36 patients with a percentage of $9.2 \%$ having ascites). Upper GIT endoscope was done to all patients for diagnosis and treatment gastro-oesophageal varices. The endoscopic findings of the patients were listed in table $(2,3)$ as follow shown Table $2 \& 3$.

Rapid urease test was done for all biopsies taken for diagnosis of H.Pylori infection and the results were as follow:

H.pylori infection was 60 patients in group A and 112 patients in group B with a total number of 172(43.8\%) (Figure 1). Gastrooesphageal variceal bleeding was seen in $31.6 \%(\mathrm{n}=60)$ of the H.Pylori infected patients and in $68.4 \%(\mathrm{n}=130)$ of the no infected patients (total number was 190 patients $)(\mathrm{P}<0.001)$. Thus, the non-bleeding patients had a higher rate of H.Pylori infection than bleeding patients with a statistically significant difference between the two studied groups as regard infection with H.Pylori (Table 4)

Table 2 Endoscopic findings of cases in group A

\begin{tabular}{llll}
\hline & Endoscopic findings & Frequency & Percent \\
\hline & Oesphageal varices(OV) & 163 & $85.80 \%$ \\
$\begin{array}{l}\text { Oesphago gastric } \\
\text { varices(OGV) }\end{array}$ & Fundal varices(FV) & 8 & $4.20 \%$ \\
& OV \& FV & 19 & $10 \%$ \\
\hline
\end{tabular}

Table 3 Endoscopic findings of cases in group B

\begin{tabular}{llll}
\hline & Endoscopic findings & Frequency & Percent \\
\hline & OV & 168 & $83.20 \%$ \\
$\begin{array}{ll}\text { Oesphago gastric } \\
\text { varices(OGV) }\end{array}$ & FV & $1 \mathrm{I}$ & $5.40 \%$ \\
& OV \& FV & 23 & $11.40 \%$ \\
\hline
\end{tabular}

Table 4 Relationship between H.Pylori infection and variceal bleeding

\begin{tabular}{lllllllll}
\hline & & \multicolumn{2}{c}{ Group A } & \multicolumn{3}{c}{ Group B } & \multirow{2}{*}{ X2 } & P \\
\cline { 3 - 6 } & & Number & $\%$ & Number & $\%$ & & \\
\hline $\begin{array}{l}\text { H pylori } \\
\text { infection }\end{array}$ & Yes & 60 & 31.6 & 112 & 55.4 & \multirow{2}{*}{22.65} & $<0.001$ \\
& No & 130 & 68.4 & 90 & 44.6 & & \\
\hline
\end{tabular}

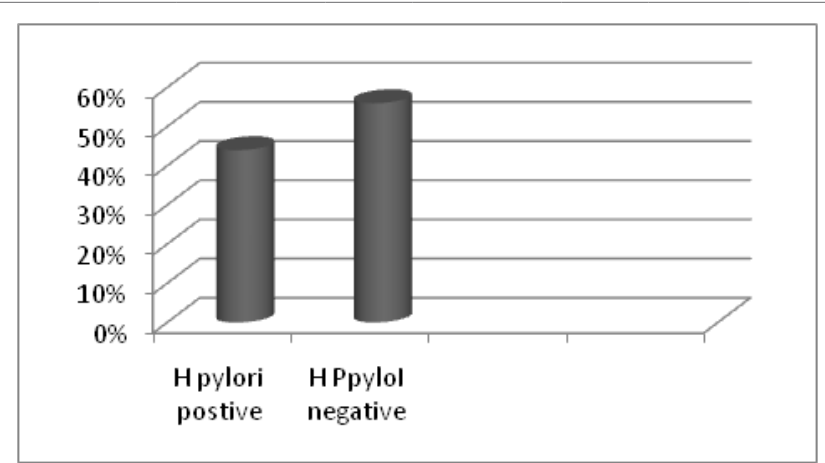

Figure I shows Prevalence of $H$. Pylori infection in all cases included in this study.

\section{Discussion}

Patients with liver cirrhosis and portal hypertension carry high risk of upper GIT bleeding. Many risk factors have been reported for bleeding from esophageal varices .Systemic risk factors such as ascites, hepatic functional reserve, HCC and endotoxemia. ${ }^{7}$ Local risk factors include esophagitis, degree of gastropathy, size of the varices, red colour sign, ${ }^{8}$ alcohol and the use of an NSAID. The hemodynamic factors as high portal pressure, ${ }^{9}$ and high intravariceal pressure with variceal wall tension. ${ }^{10}$

Portal hypertensive gastropathy include changes in mucosa of the stomach of patients with liver cirrhosis It is associated with tortuosity and dilation of the submucosal vessels, increased area of the gastric mucosa occupied by vessels and thinning of the vascular wall, and ${ }^{11}$ there is activation of growth factors and cytokines, such as 
tumor necrosis factor-alpha (TNF- $\alpha$ ) and interleukin (IL)-6. ${ }^{12}$ TNF- $\alpha$ activates endothelial nitric oxide synthase and endothelin- 1 in the gastric mucosa of patients with portal hypertension. ${ }^{13}$ Over expressed nitric oxide (NO) synthase produces an excess of NO, which induces peroxynitrite overproduction and hyperdynamic circulation. Over expression of peroxynitrite together with endothelin-1 alters gastric mucosal defense mechanism, which are impaired in portal hypertension so, gastric mucosa becomes weak and tends to be easily injured. ${ }^{14}$

H.Pylori is considered a risk factor for peptic ulcer in patients with cirrhosis . There is a significant increase in the production of inducible nitric oxide synthase (iNOS) in patients with portal hypertensive gastropathy. H.Pylori also increases expression of iNOS. ${ }^{15}$

Initially we expected that when inflammation from $H$. Pylori infection is added to the surface mucosa of varices, mucosal breaks can occur easily and become a triggering factor for bleeding varices However, the incidence of gastro-oesphageal variceal bleeding was significantly lower than expected in patients infected with H.Pylori. It may be explained by atrophic gastritis induced by H.Pylori infection which leads to decrease acid production which has harmful effect on variceal wall.

In some studies, it has been reported that the prevalence of H.Pylori infection is significantly lower in patients with gastroesophageal reflux than without. ${ }^{16}$ In addition, another report has found that H. Pylori infection inhibits reflux esophagitis by inducing atrophic gastritis. ${ }^{17}$ These data confirmed that gastric acid may be involved in esophageal bleeding because of variceal rupture. ${ }^{18}$

\section{Conclusion}

The results of our study demonstrated that H.Pylori infection was associated with low incidence of bleeding in patients with gastro esophageal varices than without and even may have a protective effect on variceal bleeding through induction of atrophic gastritis and concomitant hypoacidity.

\section{Acknowledgments}

None.

\section{Conflict of interest}

Author declares that there is no conflict of interest.

\section{References}

1. Suerbaum S, Michetti P. Helicobacter pylori infection. $N$ Engl J Med. 2002;347(15):1175-1186.

2. CoverTL, Blaser MJ. Helicobacterin health and disease. Gastroenterology. 2009;136(6):1863-1873.
3. Rosołowski R, Hartleb M, Marek T, et al. Therapeutic and prophylactic management of bleeding from oesophageal and gastric varicesrecommendations of the Working Group of the National Consultant for Gastroenterology. Prz Gastroenterol. 2014;9(2):63-68.

4. Kirchner GI, Beil W, Bleck JS, et al. Prevalence of Helicobacter pylori and occurrence of gastroduodenal lesions in patients with liver cirrhosis. Int J Clin Exp Med. 2011;4(1):26-31.

5. Cordeiro de Azevedo Conejo F, Fracassi MTM, Assef MS, et al. Elevated Gastric Antrum Erosions in Portal Hypertension Patients: Peptic Disease or Mucosal Congestion? Korean J Gastroenterol. 2017;69(5):278-282.

6. Demirturk L, Yazgan Y, Zci O, et al. The effect of Helicobacter pylori eradication on gastric juice and blood ammonia concentrations and on visual evoked potentials in cirrhosis. Helicobacter. 2001;6(40):325-330.

7. Garcia-Tsao G, Bosch J. Management of varices and variceal hemorrhage in cirrhosis. N Engl J Med. 2010;362(9):823-832.

8. Tajiri T, Yoshida H, Obara K, et al. General rules for recording endoscopic findings of esophagogastric varices. 2nd ed. Dig Endosc. 2010;22(1):1-9.

9. Iwao T, Toyonaga A, Sumino M, et al. Development of gastroesophageal varices and risk of variceal bleeding in patients with cirrhosis. Dig Endosc. 1991;3(4):485-490.

10. Mahl TC, Groszmann RJ. Pathophysiology of portal hypertension and variceal bleeding. Surg Clin North Am. 1990;70(2):251-266.

11. Abbas Z, Yakoob J, Usman MW, et al. Effect of Helicobacter pylori and its Virulence Factors on Portal Hypertensive Gastropathy and Interleukin (IL)-8, IL-10, and Tumor Necrosis Factor-alpha Levels. Saudi $J$ Gastroenterol. 2014;20(2):120-127.

12. Curvêlo LA, Brabosa W, Rhor R, et al. Underlying mechanism of portal hypertensive gastropathy in cirrhosis: A hemodynamic and morphological approach. J Gastroenterol Hepatol. 2009;24:1541-1546.

13. Kim MY, Choi H, Baik SK, et al. Portal hypertensive gastropathy: Correlation with portal hypertension and prognosis in cirrhosis. Dig Dis Sci. 2010;55(12):3561-3567.

14. Kirchner GI, Beil W, Bleck JS et al. Prevalence of Helicobacter pylori and occurrence of gastroduodenal lesions in patients with liver cirrhosis. Int J Clin Exp Med. 2011;4(1):26-31.

15. Al Mofleh IA. Does Helicobacter pylori affect portal hypertensive gastropathy? Saudi J Gastroenterol. 2007;13(2):95-97.

16. Raghunath A, Hungin AP, Wooff D, et al. Prevalence of Helicobacter pylori in patients with gastro-oesophageal reflux disease: systematic review. BMJ. 2003;326(7392):737.

17. Koike T, Ohara S, Sekine H, et al. Helicobacter pylori infection inhibits reflux esophagitis by inducing atrophic gastritis. Am J Gastroenterol. 1999;94(12):3468-3472.

18. Shephard H, Barkin JS. Omeprazole heals mucosal ulcers associated with endoscopic injection sclerotherapy. Gastrointest Endosc. 1993;39(3):474-475. 